\title{
Rotary oscillations of a micropolar fluid sphere in a bounded medium
}

\author{
K.P. Madasu ${ }^{a, *}$, M. K. Gurdatta ${ }^{a}$ \\ ${ }^{a}$ Department of Mathematics, National Institute of Technology, Raipur-492010, Chhattisgarh, India \\ Received 11 January 2017; accepted 3 May 2017
}

\begin{abstract}
The present study examines the axisymmetric rotary oscillation of a micropolar fluid sphere in concentric spherical cavity filled with Newtonian viscous fluid. A continuity of velocity components and stress together with the spin vorticity relation are used at the interface between fluid-fluid regions. The torque exerted on the micropolar fluid sphere is obtained analytically and the real and imaginary torque coefficients are presented graphically. The effect of the micropolarity parameter, viscosity ratio and spin parameter on the torque are studied numerically. In the limiting cases, the torque acting on the rotating micropolar fluid sphere and solid sphere in concentric spherical cavity are obtained from the present analysis.
\end{abstract}

(c) 2017 University of West Bohemia. All rights reserved.

Keywords: rotary oscillations, micropolar fluid, torque, spin vorticity relation

\section{Introduction}

The area of research concerning the oscillatory Stokes flows has continued to receive much attention from investigators due to its numerous applications both in engineering and science. These problems include the Brownian motion, ultra-filtration, biomechanics of blood flow and other biological or chemical phenomena [24]. The oscillating cylindrical disk viscometer which is used in measurement of fluid viscosity is the most prominent application of rotary oscillations of axisymmetric bodies [24].

The classical Navier-Stokes theory has been proved to be inadequate to describe the behavior of fluids with microstructure such as animal blood, body fluids, liquid crystals and lubricating oils etc. In the past few years there has been increasing interest in developing theories that can accurately describe the behavior of such fluids. The theory of micropolar fluids introduced by Eringen [6,7] is one of the best theories of fluids to describe the structured fluids. These fluids consist of rigid particles which can rotate with their own spins and microrotations. Micropolar fluids exhibit microscopic effects and can sustain couple stresses. There are two vectors in the micropolar fluid theory which describe the motion of the fluid; the usual velocity vector and the microrotation or spin vector. The applications of these fluids are in blood flow, lubrication problem, colloidal suspensions, liquid crystals, occurrence of turbulence, polymeric additives etc. The review article by Ariman et al. [3] and the book written by Lukaszewicz [15] provide a useful account of the applications and theory of micropolar fluids.

The oscillatory Stokes flow problem has been studied analytically and numerically by various researchers. The first study undertaken of an oscillatory flow problem was done by Stokes [22]. The author has studied the flow due to the longitudinal oscillations of a sphere

\footnotetext{
*Corresponding author. Tel.: +919 575508 447, e-mail: madaspra.maths@nitrr.ac.in. https://doi.org/10.24132/acm.2017.336
} 
in an incompressible viscous fluid. Kanwal [10] used the Stokes stream function to study the rotatory and longitudinal oscillations of various axisymmetric bodies like a sphere, a circular disk, an infinite circular cylinder, an oblate spheroid and a prolate spheroid. Lamb [14] has studied the rotatory oscillations of a sphere bounded by another concentric sphere. Tekasakul et al. [24] investigated the problem of rotatory oscillation of axisymmetric body in viscous fluid numerically for no-slip boundary condition. They evaluated the torque acting on the body. Tekasakul and Loyalka [23] extended the work of Tekasakul et al. [24] into the slip regime. They studied the effect of slip on the torque exerted on the oscillating spheres and spheroids and found that slip reduces the torque in all cases.

Lakshmana Rao and Iyengar [13] examined the rotary oscillation of a spheroid in an incompressible micropolar fluid. The rectilinear and rotary oscillation of a sphere along a diameter in micropolar fluid was studied by Lakshmana Rao and Bhujanga Rao [12]. Srinivasacharya and Iyengar [20] studied the problem of rotary oscillations of an approximate sphere in a micropolar fluid. Iyengar and Vani [9] investigated the rotary oscillatory flow of an incompressible micropolar fluid confined between two concentric spheres. They evaluated the analytical expressions for velocity and microrotation components and the couple exerted on the outer and inner spheres. The problem of an incompressible micropolar fluid flow generated by the rotary oscillations of a permeable sphere was investigated by Aparna and Murthy [2]. The axisymmetric rectilinear and rotary oscillations of a spheroidal particle in an incompressible micropolar fluid were studied by Sherief et al. [19]. They evaluated the drag and couple for prolate and oblate spheroids. Recently, the problem of the rotary oscillation of a composite sphere consisting of an impermeable sphere enclosed by a porous shell in an incompressible Newtonian fluid bounded by a concentric spherical cavity was investigated by Ashmawy [4] using the stress jump condition and the slip boundary condition.

The study of oscillations of solid bodies in an axisymmetric incompressible viscous or micropolar fluids have been attempted by many authors and the value of the couple has been evaluated. This couple is needed in designing and calibration of viscometers. Also, a sound knowledge of the movement of a single liquid drop in another immiscible liquid is required for many natural, industrial and biological processes. And there is no previous work dealing with the flow generated by the rotary oscillation of a micropolar fluid sphere in concentric spherical container containing Newtonian viscous fluid. This motivated us to investigate the present problem. The results of this study are useful in measurement of fluid viscosity and this type of studies is important in the case where oscillation occurs inside another axisymmetric body.

In this paper, we study the flow generated by the oscillatory rotational motion of a micropolar fluid sphere in Newtonian fluid bounded by concentric spherical container. The boundary conditions used at the liquid-liquid interface are the continuity of velocity components, the continuity of stress components and the spin vorticity relation. An analytic expression for the torque exerted on the micropolar fluid sphere has been obtained and its variation with various fluid parameters is studied.

\section{Formulation of the problem}

Consider the oscillatory rotational motion of an incompressible micropolar fluid sphere of radius $a$ in an incompressible Newtonian fluid bounded by concentric spherical container of radius $b$ (see Fig. 1). It is assumed that the micropolar fluid sphere is rotating about $z$-axis with angular velocity $\Omega \mathrm{e}^{\mathrm{i} \omega t}, \mathrm{i}=\sqrt{-1}$, where $\Omega$ is a constant and $\omega$ is the frequency of oscillation. 


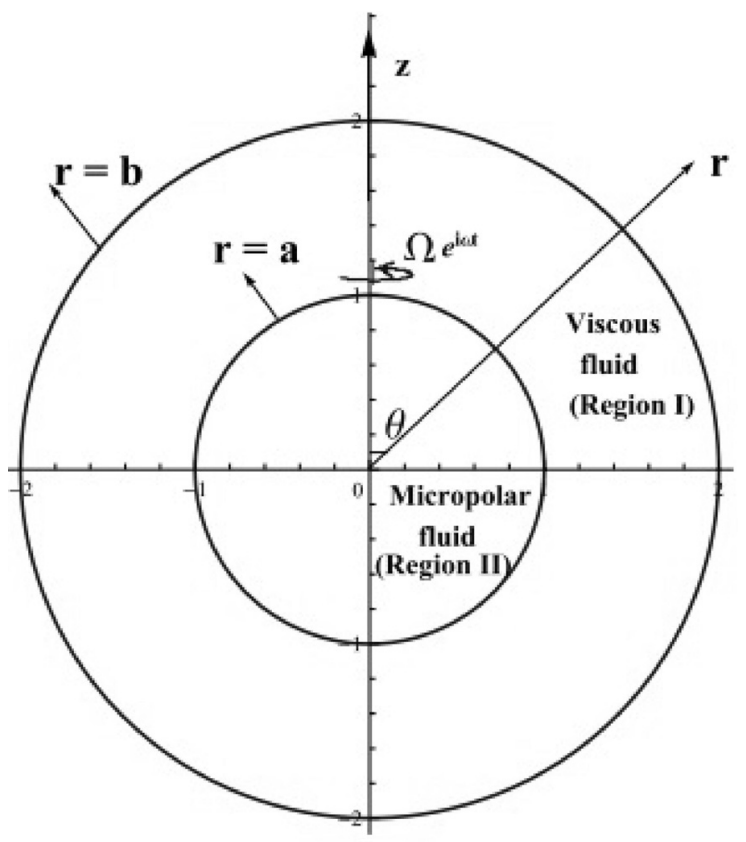

Fig. 1. Geometry of the problem

The region outside and inside the micropolar fluid sphere are denoted by regions I and II, respectively. The equations of motion governing the flow in region I are

$$
\begin{aligned}
\nabla \cdot \vec{q}^{(1)} & =0, \\
\nabla p^{(1)}+\mu_{1} \nabla \times \nabla \times \vec{q}^{(1)} & =-\rho \frac{\partial \vec{q}^{(1)}}{\partial t},
\end{aligned}
$$

where $\vec{q}^{(1)}$ is the velocity, $p^{(1)}$ is the pressure, $\mu_{1}$ is the coefficient of viscosity and $\rho$ is the fluid density.

The equations governing the unsteady flow of an incompressible micropolar fluid with the absence of body force and body couple under the Stokesian assumption in region II are given by

$$
\begin{aligned}
\nabla \cdot \vec{q}^{(2)} & =0, \\
\nabla p^{(2)}+\left(\mu_{2}+\kappa\right) \nabla \times \nabla \times \vec{q}^{(2)}-\kappa \nabla \times \vec{\nu} & =-\rho \frac{\partial \vec{q}^{(2)}}{\partial t}, \\
\kappa \nabla \times \vec{q}^{(2)}-2 \kappa \vec{\nu}-\gamma_{0} \nabla \times \nabla \times \vec{\nu}+\left(\alpha_{0}+\beta_{0}+\gamma_{0}\right) \nabla \nabla \cdot \vec{\nu} & =\rho j \frac{\partial \vec{\nu}}{\partial t},
\end{aligned}
$$

where $\vec{q}^{(2)}, \vec{\nu}, p^{(2)}$ and $\rho$ are velocity vector, microrotation vector, pressure and density of fluid, $\mu_{2}$ is the viscosity coefficient of the classical viscous fluid and $\kappa, \alpha_{0}, \beta_{0}$ and $\gamma_{0}$ are the viscosity coefficients for the micropolar fluids. The new viscosity coefficient $\kappa$ is called as micropolarity parameter. This allows us to measure the deviation of flows of micropolar fluids from that of the Navier-Stokes model. Also when this viscosity coefficient becomes zero, the conservation law of the linear momentum becomes independent of the presence of the microstructure.

The constitutive equations for the stress tensor $t_{i j}$ and the couple stress tensor $m_{i j}$ are given as

$$
\begin{aligned}
t_{i j} & =-p \delta_{i j}+\mu_{2}\left(q_{i, j}+q_{j, i}\right)+\kappa\left(q_{j, i}-\epsilon_{i j m} \nu_{m}\right), \\
m_{i j} & =\alpha_{0} \nu_{m, m} \delta_{i j}+\beta_{0} \nu_{i, j}+\gamma_{0} \nu_{j, i},
\end{aligned}
$$


where the comma denotes the partial differentiation, $\delta_{i j}$ and $\epsilon_{i j m}$ are the Kronecker delta and the alternating tensor.

Let $(r, \theta, \phi)$ denote a spherical polar co-ordinate system with origin at the center of a micropolar fluid sphere $r=a$. The motion is axially symmetric about $z$-axis, thus all the quantities are independent of $\phi$. We then choose the velocity vectors and pressures in both the regions and microrotation vector in region II as

$$
\begin{aligned}
\vec{q}^{(i)} & =q_{\phi}^{(i)}(r, \theta) \mathrm{e}^{\mathrm{i} \omega t} \vec{e}_{\phi}, \quad i=1,2, \\
\vec{\nu} & =\nu_{r}(r, \theta) \mathrm{e}^{\mathrm{i} \omega t} \vec{e}_{r}+\nu_{\theta}(r, \theta) \mathrm{e}^{\mathrm{i} \omega t} \vec{e}_{\theta}, \\
p^{(i)} & =p^{(i)} \mathrm{e}^{\mathrm{i} \omega t}, \quad i=1,2 .
\end{aligned}
$$

Assume that $\operatorname{div} \vec{\nu}=g(r, \theta) \mathrm{e}^{\mathrm{i} \omega t}$ and $\operatorname{curl} \vec{\nu}=h(r, \theta) \mathrm{e}^{\mathrm{i} \omega t} \vec{e}_{\phi}$.

Therefore, the field equations in this case reduce to:

For the viscous fluid region $a \leq r \leq b$,

$$
\begin{aligned}
\frac{\partial p^{(1)}}{\partial r} & =0, \quad \frac{\partial p^{(1)}}{\partial \theta}=0, \\
\left(L-l^{2}\right) q_{\phi}^{(1)} & =0,
\end{aligned}
$$

where

$$
l^{2}=\frac{i \rho \omega a^{2}}{\mu_{1}}
$$

and for the micropolar fluid region $r \leq a$,

$$
\begin{aligned}
\frac{\partial p^{(2)}}{\partial r} & =0, \quad \frac{\partial p^{(2)}}{\partial \theta}=0 \\
\left(L-\frac{i \rho \omega}{\mu_{2}+\kappa}\right) q_{\phi}^{(2)} & =\frac{-\kappa}{\mu_{2}+\kappa} h(r, \theta) \\
\nu_{r} & =\frac{1}{c^{2}} \frac{\partial g}{\partial r}-\frac{\gamma_{0}}{2 \kappa+i j \rho \omega} \frac{1}{r}\left(\frac{\partial h}{\partial \theta}+h \cot \theta\right)+ \\
& \frac{\kappa}{2 \kappa+i j \rho \omega} \frac{1}{r}\left(\frac{\partial q_{\phi}^{(2)}}{\partial \theta}+q_{\phi}^{(2)} \cot \theta\right) \\
\nu_{\theta} & =\frac{1}{c^{2}} \frac{1}{r} \frac{\partial g}{\partial \theta}+\frac{\gamma_{0}}{2 \kappa+i j \rho \omega}\left(\frac{\partial h}{\partial r}+\frac{h}{r}\right)-\frac{\kappa}{2 \kappa+i j \rho \omega}\left(\frac{\partial q_{\phi}^{(2)}}{\partial r}+\frac{q_{\phi}^{(2)}}{r}\right) .
\end{aligned}
$$

From (15) and (16), we have

$$
\left(\nabla^{2}-c^{2}\right) g=0
$$

Also

$$
\left(L-\frac{2 \kappa+i j \rho \omega}{\gamma_{0}}\right) h=\frac{\kappa}{\gamma_{0}} L q_{\phi}^{(2)}
$$

where

$$
\begin{aligned}
c^{2} & =\frac{(2 \kappa+i j \rho \omega) a^{2}}{\alpha_{0}+\beta_{0}+\gamma_{0}}, \\
L & =\nabla^{2}-\frac{1}{r^{2} \sin ^{2} \theta}, \quad \nabla^{2}=\frac{\partial^{2}}{\partial r^{2}}+\frac{2}{r} \frac{\partial}{\partial r}+\frac{1}{r^{2}} \frac{\partial^{2}}{\partial \theta^{2}}+\frac{\cot \theta}{r^{2}} \frac{\partial}{\partial \theta} .
\end{aligned}
$$


Using (14) and (18) we have

$$
\left[L^{2}-\left(\frac{\kappa\left(2 \mu_{2}+\kappa\right)+i \rho \omega\left(\gamma_{0}+j \mu_{2}+j \kappa\right)}{\gamma_{0}\left(\mu_{2}+\kappa\right)}\right) L+\frac{\rho \omega(2 \kappa i-j \rho \omega)}{\gamma_{0}\left(\mu_{2}+\kappa\right)}\right] q_{\phi}^{(2)}=0 .
$$

This equation can be rewritten as

$$
\left(L-m^{2}\right)\left(L-n^{2}\right) q_{\phi}^{(2)}=0,
$$

where

$$
m^{2}+n^{2}=\frac{\left(\kappa\left(2 \mu_{2}+\kappa\right)+i \rho \omega\left(\gamma_{0}+j \mu_{2}+j \kappa\right)\right) a^{2}}{\gamma_{0}\left(\mu_{2}+\kappa\right)}
$$

and

$$
m^{2} n^{2}=\frac{\rho \omega(2 \kappa i-j \rho \omega) a^{4}}{\gamma_{0}\left(\mu_{2}+\kappa\right)}
$$

\section{Boundary conditions}

To obtain the complete solution of the problem, we assume the continuity of velocity components. Also we assume as in the classical case [8], that the equilibrium theory of interfacial tension is applicable to our problem. This means that the presence of interfacial tension only produces a discontinuity in the normal stresses and does not in any way affect the tangential stresses. The latter is therefore continuous across the surface of the fluid sphere. Hence, continuity of tangential stresses is applied at the interface. There in no general agreement for the microrotation boundary condition in the literature. Migun [17] investigated various types of boundary conditions for microrotation. Aero et al. [1] proposed a dynamic boundary condition for microrotation which states that the microrotation is proportional to the couple stress at the boundary. Condiff and Dahler [5] suggested a spin vorticity kinematic boundary condition which states that the microrotation is proportional to the vorticity. In the present study we apply the spin vorticity relationship at the boundary. These conditions are physically realistic and mathematically consistent $[5,8,9,16]$.

The boundary conditions on the surface $r=a$ are

i. Continuity of velocity components

$$
q_{\phi}^{(1)}=q_{\phi}^{(2)}
$$

ii. Continuity of tangential stress components

$$
t_{r \phi}^{(1)}=t_{r \phi}^{(2)} .
$$

iii. Spin vorticity relation

$$
\begin{aligned}
& \nu_{r}=\frac{s}{2 r}\left[\frac{\partial q_{\phi}^{(1)}}{\partial \theta}+q_{\phi}^{(1)} \cot \theta\right], \\
& \nu_{\theta}=-\frac{s}{2}\left[\frac{\partial q_{\phi}^{(1)}}{\partial r}+\frac{q_{\phi}^{(1)}}{r}\right],
\end{aligned}
$$


where $s$ is a spin parameter that varies from 0 to 1 . The value $s=0$ (no spin) corresponds to the case where microelements close to a solid boundary are unable to rotate, whereas the value $s=1$ corresponds to the case where the microrotation is equal to the fluid vorticity at the boundary. This parameter is assumed to depend only on the nature of the fluids.

The boundary condition on the surface $r=b$ is

iv. On the container surface

$$
q_{\phi}^{(1)}=-\Omega r \sin \theta
$$

\section{Solution of the problem}

Using the method of separation of variables, the solution of (12) and (17) are given respectively

$$
\begin{aligned}
q_{\phi}^{(1)} & =\left[r^{-1 / 2} K_{3 / 2}(l r) c_{1}+r^{-1 / 2} I_{3 / 2}(l r) d_{1}\right] \sin \theta, \\
g(r, \theta) & =r^{-1 / 2} I_{3 / 2}(c r) e_{1} \cos \theta .
\end{aligned}
$$

The solution of (20) is obtained by superposing the solutions of

$$
\begin{gathered}
\left(L-m^{2}\right) q_{\phi}^{(2)}=0, \\
\left(L-n^{2}\right) q_{\phi}^{(2)}=0,
\end{gathered}
$$

and again using the method of separation of variables, we get

$$
q_{\phi}^{(2)}=\left[r^{-1 / 2} I_{3 / 2}(m r) a_{1}+r^{-1 / 2} I_{3 / 2}(n r) b_{1}\right] \sin \theta,
$$

where $a_{1}, b_{1}, c_{1}, d_{1}$ and $e_{1}$ are arbitrary constants to be evaluated by applying the boundary conditions (21)-(25). Using (14) and (30) we get

$$
\begin{aligned}
h(r, \theta)= & -\left[a_{1} r^{-1 / 2}\left(\frac{m^{2}\left(\mu_{2}+\kappa\right)-i \rho \omega}{\kappa}\right) I_{3 / 2}(m r)+\right. \\
& \left.b_{1} r^{-1 / 2}\left(\frac{n^{2}\left(\mu_{2}+\kappa\right)-i \rho \omega}{\kappa}\right) I_{3 / 2}(n r)\right] \sin \theta .
\end{aligned}
$$

Thus, using the expressions for $g, h$ and $q_{\phi}^{(2)}$ in (15) and (16), the expressions for $\nu_{r}$ and $\nu_{\theta}$ are obtained as

$$
\begin{aligned}
\nu_{r}= & {\left[-\frac{1}{c^{2}} \frac{1}{r^{3 / 2}}\left(2 I_{3 / 2}(c r)-c r I_{1 / 2}(c r)\right) e_{1}+\right.} \\
& \left.\frac{2}{r^{3 / 2}}\left(a_{1} I_{3 / 2}(m r) A_{m}+b_{1} I_{3 / 2}(n r) A_{n}\right)\right] \cos \theta \\
\nu_{\theta}= & {\left[-\frac{1}{c^{2}} \frac{1}{r^{3 / 2}} I_{3 / 2}(c r) e_{1}+\frac{1}{r^{3 / 2}} a_{1} A_{m}\left(I_{3 / 2}(m r)-m r I_{1 / 2}(m r)\right)+\right.} \\
& \left.\frac{1}{r^{3 / 2}} b_{1} A_{n}\left(I_{3 / 2}(n r)-n r I_{1 / 2}(n r)\right)\right] \sin \theta,
\end{aligned}
$$

where

and

$$
A_{m}=\frac{\gamma_{0} m^{2}\left(\mu_{2}+\kappa\right)+\kappa^{2}-\gamma_{0} i \rho \omega}{\kappa(2 \kappa+i j \rho \omega)}
$$

$$
A_{n}=\frac{\gamma_{0} n^{2}\left(\mu_{2}+\kappa\right)+\kappa^{2}-\gamma_{0} i \rho \omega}{\kappa(2 \kappa+i j \rho \omega)} .
$$




\section{Torque on the body}

The torque exerted by the Newtonian fluid on micropolar fluid sphere is given by

$$
T=\left.2 \pi a^{3} \int_{0}^{\pi} t_{r \phi}^{(1)}\right|_{r=1} \sin ^{2} \theta \mathrm{d} \theta
$$

where the shear stress of the Newtonian fluid (region I) is given by

$$
t_{r \phi}^{(1)}=\mu_{1}\left[\frac{\partial q_{\phi}^{(1)}}{\partial r}-\frac{q_{\phi}^{(1)}}{r}\right] \mathrm{e}^{\mathrm{i} \omega t} .
$$

After some calculations, (34) reduces to

$$
\begin{aligned}
T= & \frac{8}{3} \pi \mu_{1} \Omega a^{3}\left(-K^{\prime}-i K\right) \mathrm{e}^{\mathrm{i} \omega t}= \\
& -\frac{8}{3} \pi \mu_{1} \Omega a^{3}\left[c_{1}\left(3 K_{3 / 2}(l)+l K_{1 / 2}(l)\right)+d_{1}\left(3 I_{3 / 2}(l)-l I_{1 / 2}(l)\right)\right] \mathrm{e}^{\mathrm{i} \omega t},
\end{aligned}
$$

where $K^{\prime}$ and $K$ are the real and imaginary torque coefficients, respectively. Thus, the real and imaginary parts of the torque are, respectively, given by

$$
\Re T=\frac{8}{3} \pi \mu_{1} \Omega a^{3}\left(K \sin \omega t-K^{\prime} \cos \omega t\right), \quad \Im T=-\frac{8}{3} \pi \mu_{1} \Omega a^{3}\left(K \cos \omega t+K^{\prime} \sin \omega t\right) .
$$

\subsection{Special cases}

i. The case of slow steady rotation of a micropolar fluid sphere is obtained from the above analysis by allowing the period of oscillation $2 \pi / \omega$ tend to infinity. Using

$$
\lim _{\omega \rightarrow 0}\left(m^{2}+n^{2}\right)=m_{1}^{2}, \quad \lim _{\omega \rightarrow 0}\left(m^{2} n^{2}\right)=0,
$$

where

$$
m_{1}^{2}=\frac{\kappa\left(2 \mu_{2}+\kappa\right) a^{2}}{\gamma_{0}\left(\mu_{2}+\kappa\right)}
$$

So we take $m=m_{1}, n=0$ and $l=0$. We get

$$
T=-8 \pi \mu_{1} \Omega a^{3} \chi(1-s) \Delta_{1} / \Delta_{2},
$$

where

$$
\begin{aligned}
\Delta_{1}= & \left(3(3+2 \chi) I_{3 / 2}(m)-m(1+\chi) I_{1 / 2}(m)\right) I_{3 / 2}(c)-c(2+\chi) I_{3 / 2}(m) I_{1 / 2}(c), \\
\Delta_{2}= & I_{3 / 2}(c)\left(2 l(1+\chi) I_{1 / 2}(l)\left(3 \lambda(2+\chi)-\chi\left(1-\eta^{3}\right)(1-s)\right)+3 I_{3 / 2}(l) \chi\right. \\
& \left.\left(4 \chi+6-s \chi-3 \lambda_{1}(2+\chi)-2 \eta^{3}(1-s)(3+2 \chi)\right)\right)+c(2+\chi) I_{1 / 2}(c) \\
& \left(-3 \lambda l I_{1 / 2}(l)(1+\chi)+I_{3 / 2}(l)\left(\lambda(3+6 \chi)-\left(s+2-2 \eta^{3}(1-s)\right) \chi\right)\right), \\
m^{2}= & \frac{\kappa\left(2 \mu_{2}+\kappa\right) a^{2}}{\gamma_{0}\left(\mu_{2}+\kappa\right)}=\frac{\kappa(2+\chi) a^{2}}{\gamma_{0}(1+\chi)}, \quad c^{2}=\frac{2 \kappa a^{2}}{\alpha_{0}+\beta_{0}+\gamma_{0}}, \quad \chi=\frac{\kappa}{\mu_{2}}, \\
\lambda_{1}= & \frac{2 \sigma}{2+\chi}, \quad \sigma=\frac{\mu_{1}}{\mu_{2}}, \quad \eta=\frac{a}{b} .
\end{aligned}
$$

This result agrees with the result previously obtained by Madasu and Gurdatta [16]. 
ii. When $s \rightarrow 0$ and $\sigma \rightarrow 0$, the expression for the torque in case of rotary oscillation of a solid sphere in a concentric spherical cavity of viscous fluid is obtained as

$$
T=\frac{8 \pi \mu_{1} a^{3} \Omega\left(I_{3 / 2}(l) K_{1 / 2}(l)+I_{1 / 2}(l) K_{3 / 2}(l)\right) l \eta^{-3 / 2}}{3\left(I_{3 / 2}(l) K_{3 / 2}\left(l \eta^{-1}\right)-I_{3 / 2}\left(l \eta^{-1}\right) K_{3 / 2}(l)\right)} \mathrm{e}^{\mathrm{i} \omega t} .
$$

This agrees with the result obtained by Ashmawy [4] in case of no slip condition.

iii. When $s \rightarrow 0$ and $\chi \rightarrow 0$, the expression for the torque in case of rotary oscillation of a viscous fluid sphere in another immiscible viscous fluid is obtained as

$$
T=8 \pi \mu_{1} a^{3} \Omega\left[\frac{\left(I_{3 / 2}(l) K_{1 / 2}(l)+I_{1 / 2}(l) K_{3 / 2}(l)\right) l \eta^{-3 / 2}}{\Delta_{3}}\right] \mathrm{e}^{\mathrm{i} \omega t},
$$

where

$$
\begin{gathered}
\Delta_{3}=3(1-\lambda)\left(I_{3 / 2}(l) K_{3 / 2}\left(l \eta^{-1}\right)-I_{3 / 2}\left(l \eta^{-1}\right) K_{3 / 2}(l)+\right. \\
\left.l \lambda\left(I_{1 / 2}(l) K_{3 / 2}\left(l \eta^{-1}\right)+I_{3 / 2}\left(l \eta^{-1}\right) K_{1 / 2}(l)\right)\right) .
\end{gathered}
$$

iv. When $\omega \rightarrow 0$ in (42), the expression for the torque in case of slow steady rotation of a solid sphere in spherical container is obtained as

$$
T=-\frac{8 \pi \mu_{1} a^{3} \Omega}{1-\eta^{3}}
$$

This agrees with the result previously obtained by Keh and Lu [11], Saad [18], Srinivasacharya and Krishna Prasad [21].

\section{Results and Discussion}

The variations of the torque coefficients $K^{\prime}$ and $K$ with the ratio of viscosities between internal and external fluid $\sigma$ and frequency parameter $\omega_{1}=\rho \omega a^{2} / \mu_{2}$ are shown in Figs. 2-7 for different values of the spin parameter $s$, micropolarity parameter $\chi$ and separation parameter $\eta$. During

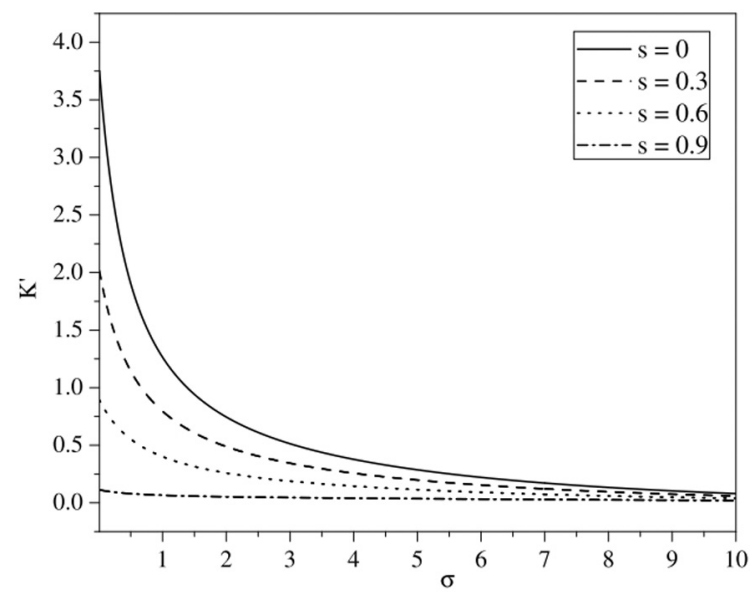

(a) Variation of $K^{\prime}$ with $\sigma$

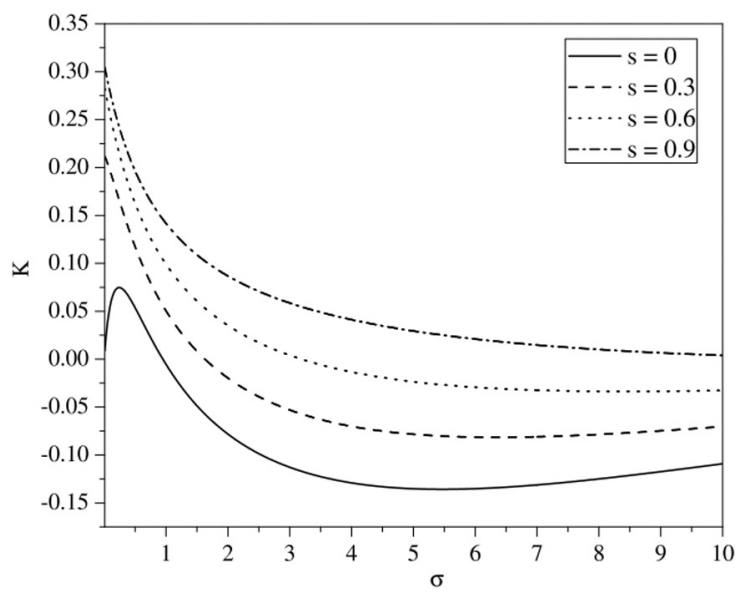

(b) Variation of $K$ with $\sigma$

Fig. 2. Variation of the torque coefficients versus viscosity ratio $\sigma$ for different values of $s$ with $\eta=0.6$ and $\chi=2$ 


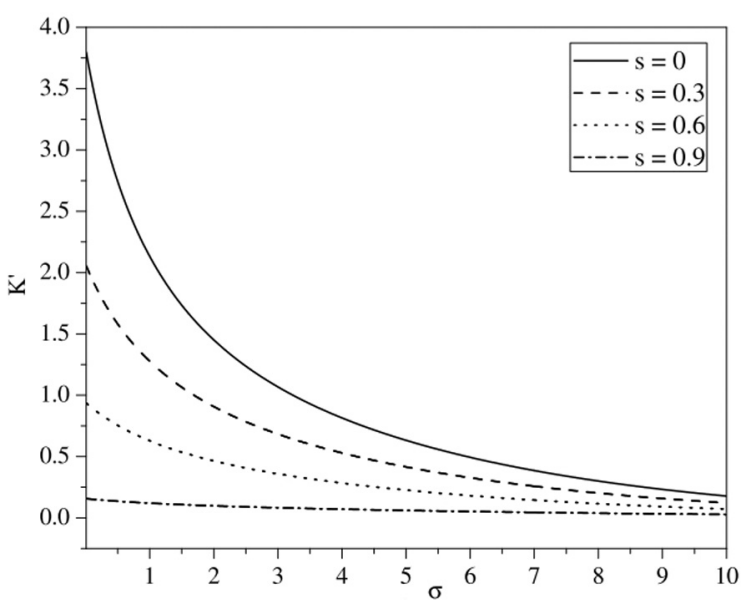

(a) Variation of $K^{\prime}$ with $\sigma$

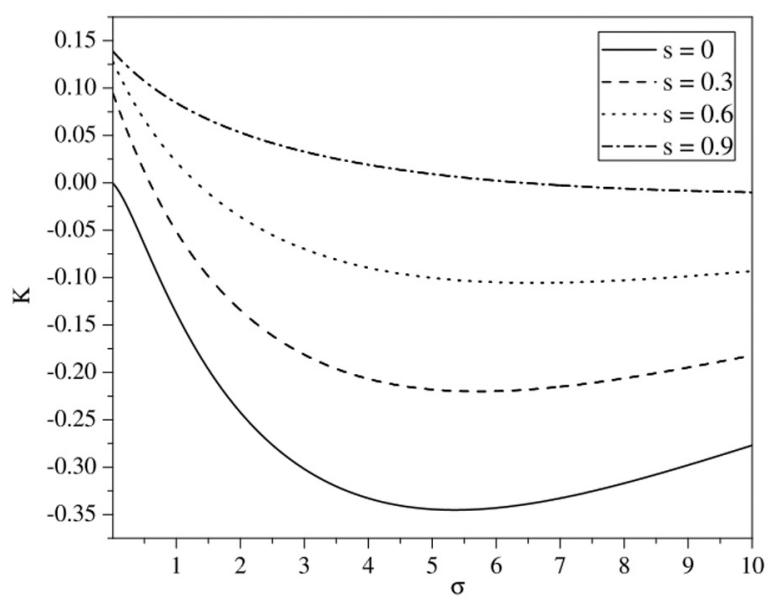

(b) Variation of $K$ with $\sigma$

Fig. 3. Variation of the torque coefficients versus viscosity ratio $\sigma$ for different values of $s$ with $\eta=0.6$ and $\chi=5$

numerical calculations we assumed the value of $j / a^{2}=0.2, \alpha_{0} /\left(\mu_{2} a^{2}\right)=0.1, \beta_{0} /\left(\mu_{2} a^{2}\right)=0.2$, $\gamma_{0} /\left(\mu_{2} a^{2}\right)=0.3$ and $\omega_{1}=1$.

Figs. 2 and 3 illustrate the graphical representation of $K^{\prime}$ and $K$ with viscosity ratio $\sigma$ for different values of $s$ with $\eta=0.6$ for the case of $\chi=2$ and $\chi=5$, respectively. The torque coefficient $K^{\prime}$ is greater for no spin condition, whereas the torque coefficient $K$ is smaller for no spin condition. It can also be perceived from the figures that the torque coefficient $K^{\prime}$ reaches a constant value as $\sigma$ tends to infinity. As $\sigma \rightarrow 0$, the problem reduces to the rotary oscillation of solid sphere in concentric spherical container.

The influence of micropolarity parameter on the torque coefficients $K^{\prime}$ and $K$ are shown in Figs. 4 and 5. When the micropolarity parameter $\chi \rightarrow 0$, both the fluids (in the container and in the cavity) are Newtonian and the torque acting on the viscous fluid sphere rotating steadily in concentric spherical container is obtained by taking the period of oscillation $2 \pi / \omega$ tend to infinity. In this case the torque exerted on the fluid sphere is zero. These figures indicates that the

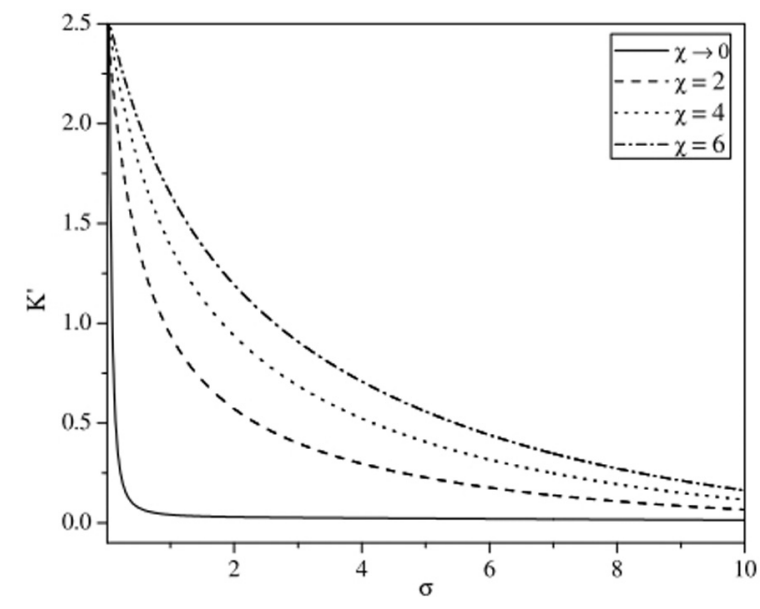

(a) Variation of $K^{\prime}$ with $\sigma$

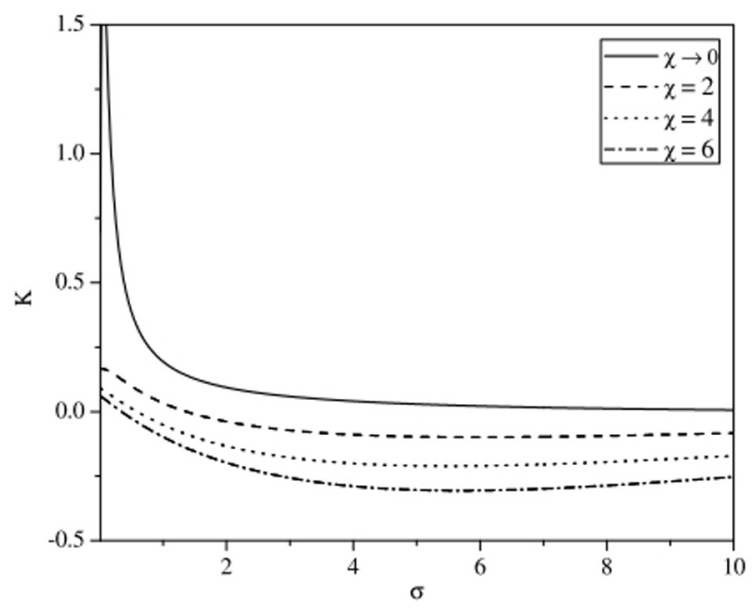

(b) Variation of $K$ with $\sigma$

Fig. 4. Variation of the torque coefficients versus viscosity ratio $\sigma$ for different values of $\chi$ with $\eta=0.6$ and $s=0.2$ 


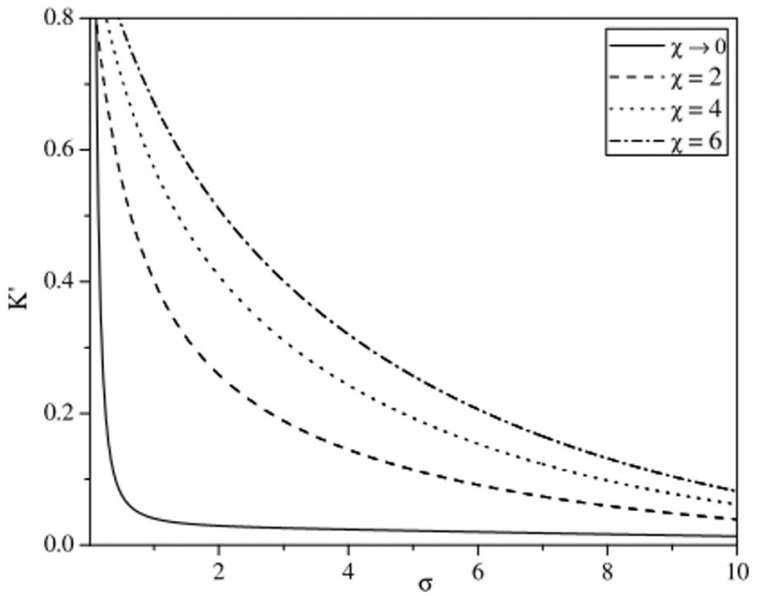

(a) Variation of $K^{\prime}$ with $\sigma$

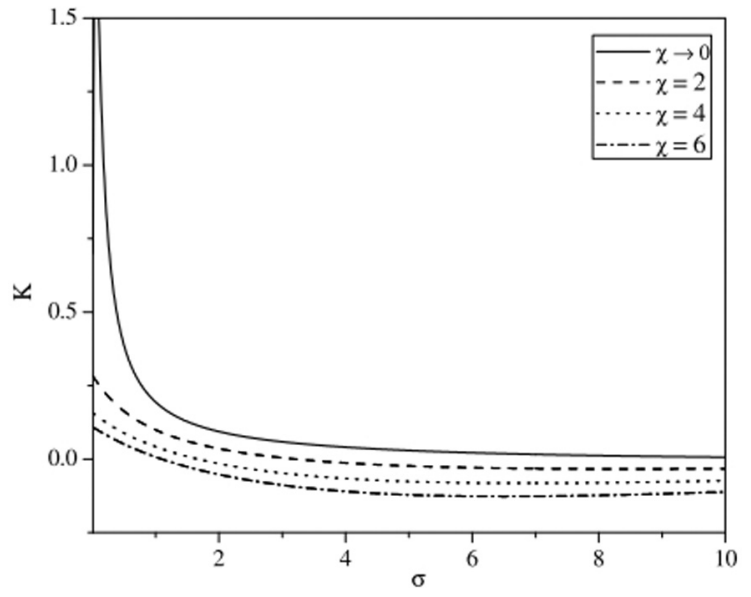

(b) Variation of $K$ with $\sigma$

Fig. 5. Variation of the torque coefficients versus viscosity ratio $\sigma$ for different values of $\chi$ with $\eta=0.6$ and $s=0.6$

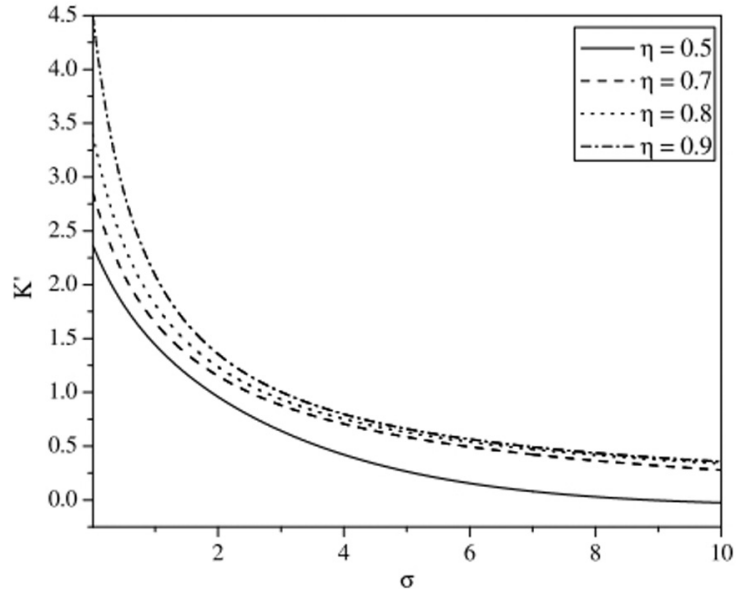

(a) Variation of $K^{\prime}$ with $\sigma$

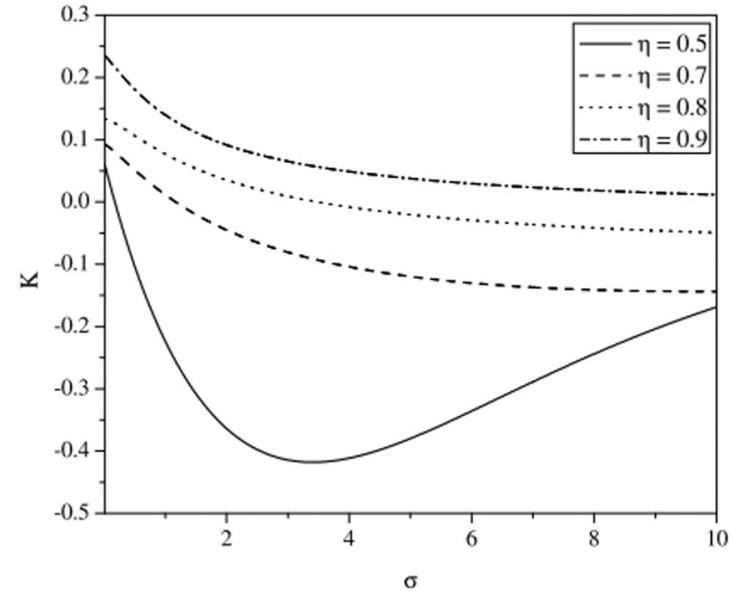

(b) Variation of $K$ with $\sigma$

Fig. 6. Variation of the torque coefficients versus viscosity ratio $\sigma$ for different values of $\eta$ with $s=0.6$ and $\chi=5$

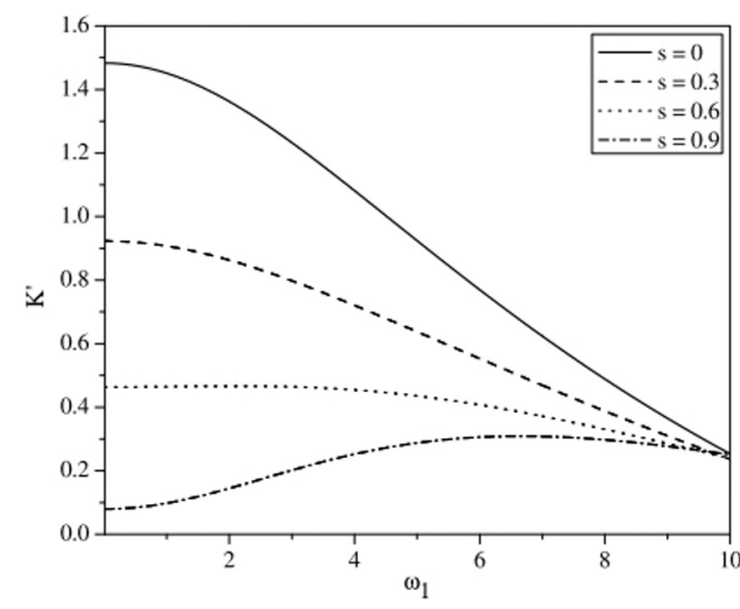

(a) Variation of $K^{\prime}$ with $\omega_{1}$

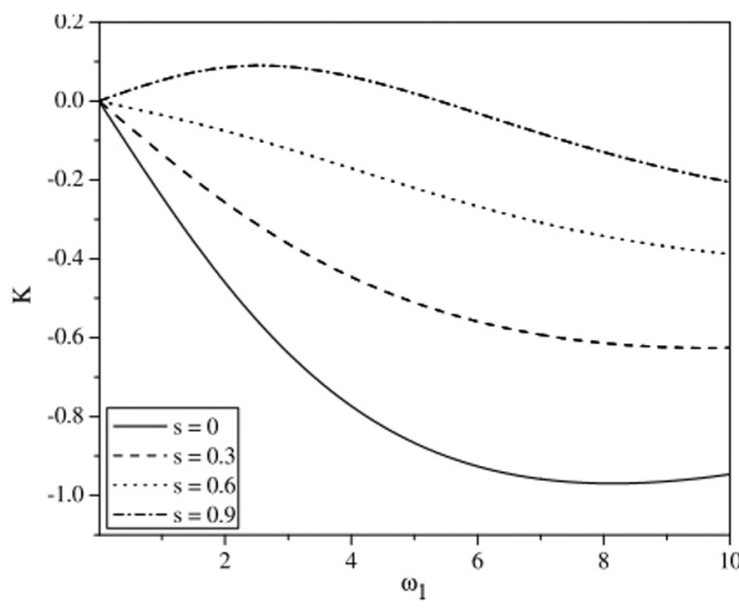

(b) Variation of $K$ with $\omega_{1}$

Fig. 7. Variation of the torque coefficients versus frequency parameter $\omega_{1}$ for different values of $s$ with $\eta=0.6, \sigma=2$, and $\chi=5$ 
torque coefficient $K^{\prime}$ of the classical viscous fluid is smaller than that of micropolar fluid. Where as the torque coefficient $K$ of the classical viscous fluid is larger than that of micropolar fluid. Also, torque coefficient decreases with increasing viscosity ratio. Fig. 6 depicts the variation of torque coefficients versus viscosity ratio $\sigma$ for different values of the separation parameter $\eta$. It can be seen from the figure that the torque coefficients decreases as viscosity ratio increases and increases with the increase of the separation parameter. Fig. 7 shows the graphical representation of torque coefficients with frequency of the oscillations $\omega_{1}$. It indicates that the torque coefficients decreases with the increase of the frequency parameter.

\section{Conclusions}

In this paper, we presented the analytical solution for the rotary oscillations of a micropolar fluid sphere in a concentric spherical cavity containing Newtonian fluid. The explicit expressions of flow fields are determined by applying the boundary conditions at the container and cavity surfaces. An expression for the torque acting on the micropolar fluid sphere is obtained in terms of two real parameters $K$ and $K^{\prime}$. Some well known results reported in the literature are also found from the present problem in the limiting cases. The torque acting on the rotary oscillating sphere decreases as the viscosity ratio increases. The real torque coefficient increases with increase in micropolarity parameter, but reverse effect is seen for imaginary torque coefficient. The effect of spin parameter on torque is also studied. It is found that the increase in spin parameter results in decrease of real torque coefficient and an increase of imaginary torque coefficient. We also found that the torque coefficient $K^{\prime}$ of a micropolar fluid is larger than that of classical fluid while the torque coefficient $K$ of a micropolar fluid is smaller than that of classical fluid.

\section{A Appendix}

Applying boundary conditions (21)-(25) on (26), (30), (32) and (33) gives the following algebraic system of equations for the determination of the arbitrary constants $a_{1}, b_{1}, c_{1}, d_{1}$ and $e_{1}$ :

$$
\begin{aligned}
{\left[a_{1} T_{4}+b_{1} T_{6}-c_{1} S_{2}-d_{1} T_{2}\right] P_{1}^{1}(\zeta) } & =0 \\
{\left[a_{1}\left(2 T_{4}-m T_{3}\right)+b_{1}\left(2 T_{6}-n T_{5}\right)+a_{1} N_{2} T_{4}+b_{1} N_{2} T_{6}+c^{-2} e_{1} N_{1} T_{8}-\right.} & \\
-a_{1} N_{1} A_{m}\left(T_{4}-m T_{3}\right)-b_{1} N_{1} A_{n}\left(T_{6}-n T_{5}\right)- & =0 \\
\left.\lambda c_{1}\left(3 S_{2}+l S_{1}\right)-\lambda d_{1}\left(3 T_{2}-l T_{1}\right)\right] P_{1}^{1}(\zeta) & =0 \\
{\left[2 a_{1} A_{m} T_{4}+2 b_{1} A_{n} T_{6}+c^{-2} e_{1} w_{2}-s c_{1} S_{2}-s d_{1} T_{2}\right] P_{1}^{1}(\zeta) } & =0 \\
{\left[a_{1} A_{m}\left(T_{4}-m T_{3}\right)+b_{1} A_{n}\left(T_{6}-n T_{5}\right)-c^{-2} e_{1} T_{8}-\right.} & \\
\left.\frac{s}{2} c_{1}\left(S_{2}+l S_{1}\right)-\frac{s}{2} d_{1}\left(T_{2}-l T_{1}\right)\right] P_{1}^{1}(\zeta) & =0, \\
{\left[c_{1} \eta^{1 / 2} S_{3}+d_{1} \eta^{1 / 2} T_{9}+\eta^{-1}\right] P_{1}^{1}(\zeta) } & =0 .
\end{aligned}
$$

Solving this algebraic system of equations, we get the expressions for $a_{1}, b_{1}, c_{1}, d_{1}$ and $e_{1}$

$$
\begin{aligned}
a_{1}= & w_{1}\left[c l\left(2 A_{n} \lambda+s\left(-2+A_{n} N_{1}-N_{2}+2 \lambda\right)\right) T_{6} T_{7}+l s\left(4+2 N_{2}-N_{1} s-6 \lambda\right) T_{6} T_{8}+\right. \\
& \left.l\left(s-A_{n} N_{1} s-2 A_{n} \lambda\right) c T_{5}\left(c T_{7}-2 T_{8}\right)\right] / \Delta, \\
b_{1}= & w_{1}\left[c l\left(2 A_{m} \lambda+s\left(-2+A_{m} N_{1}-N_{2}+2 \lambda\right)\right) T_{4} T_{7}+l s\left(4+2-N_{2} s-6 \lambda\right) T_{4} T_{8}+\right. \\
& \left.l\left(s-A_{m} N_{1} s-2 A_{m} \lambda\right) m T_{3}\left(c T_{7}-2 T_{8}\right)\right] / \Delta,
\end{aligned}
$$




$$
\begin{aligned}
c_{1}= & {\left[\left(4+2 N_{2}-N_{1} s-6 \lambda\right) w_{2} w_{3} T_{2}+\left(-l s m+A_{m} l\left(N_{1} s+2 \lambda\right) m\right) T_{1} T_{3} T_{6} w_{2}+\right.} \\
& \left(l s n-A_{n} l\left(N_{1} s+2 \lambda\right) n\right) T_{1} T_{4} T_{5} w_{2}+2 m n T_{2} T_{3} T_{5}\left(A_{n}-A_{m}\right) w_{2}-2 c T_{2} T_{7} w_{4}+ \\
& c s T_{2} T_{7} w_{5}+c\left(4+2 N_{2}-N_{1} s-6 \lambda\right) T_{2} T_{7} T_{4} T_{6}\left(A_{n}-A_{m}\right)+c l\left(N_{1} s+2 \lambda\right) \\
& \left.T_{1} T_{7} T_{4} T_{6}\left(A_{m}-A_{n}\right)\right] / \Delta, \\
d_{1}= & {\left[\left(4+2 N_{2}-N_{1} s-6 \lambda\right) w_{2} w_{3} S_{2}+\left(l s m-A_{m} l\left(N_{1} s+2 \lambda\right) m\right) S_{1} T_{3} T_{6} w_{2}+\right.} \\
& \left(-l s n+A_{n} l\left(N_{1} s+2 \lambda\right) n\right) S_{1} T_{4} T_{5} w_{2}+2 m n S_{2} T_{3} T_{5}\left(A_{n}-A_{m}\right) w_{2}-2 c S_{2} T_{7} w_{4}+ \\
& c s S_{2} T_{7} w_{5}-c\left(4+2 N_{2}-N_{1} s-6 \lambda\right) S_{2} T_{7} T_{4} T_{6}\left(A_{n}-A_{m}\right)+ \\
& \left.c l\left(N_{1} s+2 \lambda\right) S_{1} T_{7} T_{4} T_{6}\left(A_{m}-A_{n}\right)\right] / \Delta, \\
e_{1}= & -w_{1} c^{2} l\left[s\left(4+2 N_{2}-N_{1} s-6 \lambda\right)\left(A_{n}-A_{m}\right) T_{4} T_{6}+\left(A_{m}\left(2 A_{n}-s\right)\left(N_{1} s+2 \lambda\right) m+\right.\right. \\
& \left.\left.\left.s\left(-2 A_{n} m+s m\right)\right) T_{3} T_{6}+\left(s-A_{n} N_{1} s-2 A_{n} \lambda\right)\left(2 A_{m}-s\right) n T_{4} T_{5}\right)\right] / \Delta,
\end{aligned}
$$

where

$$
\begin{gathered}
\Delta=\eta^{3 / 2}\left[\operatorname{cl}\left(N_{1} s+2 \lambda\right)\left(A_{m}-A_{n}\right) T_{4} T_{6} T_{7} w_{6}+c\left(4+2 N_{2}-N_{1} s-6 \lambda\right) \times\right. \\
\left(A_{m}-A_{n}\right) T_{4} T_{6} T_{7} w_{7}+s l w_{2} w_{5} w_{6}-l\left(N_{1} s+2 \lambda\right) w_{2} w_{3} w_{6}+2 c T_{7} w_{4} w_{7}- \\
\left.-c s T_{7} w_{5} w_{7}-\left(4+2 N_{2}-N_{1} s-6 \lambda\right) w_{2} w_{3} w_{7}+2 m n\left(A_{m}-A_{n}\right) T_{3} T_{5} w_{2} w_{7}\right], \\
\lambda=\frac{\sigma}{1+\chi}, \quad N_{1}=\frac{\chi}{1+\chi}, \quad N_{2}=\frac{1}{1+\chi}, \quad \eta=\frac{a}{b}
\end{gathered}
$$

with $\sigma=\frac{\mu_{1}}{\mu_{2}}$ is the classical ratio of viscosities between the internal and external fluids.

$$
\begin{aligned}
& w_{1}=T_{2} S_{1}+T_{1} S_{2}, \quad w_{2}=c T_{7}-2 T_{8}, \\
& w_{3}=m A_{m} T_{3} T_{6}-n A_{n} T_{5} T_{4}, \quad w_{4}=m A_{n} T_{3} T_{6}-n A_{m} T_{5} T_{4}, \\
& w_{5}=m T_{3} T_{6}-n T_{5} T_{4}, \quad w_{6}=T_{9} S_{1}+T_{1} S_{3}, \\
& w_{7}=T_{2} S_{3}-T_{9} S_{2}, \\
& S_{1}=K_{1 / 2}(l), \quad S_{2}=K_{3 / 2}(l), \quad S_{3}=K_{3 / 2}\left(l \eta^{-1}\right), \\
& T_{1}=I_{1 / 2}(l), \quad T_{2}=I_{3 / 2}(l), \quad T_{3}=I_{1 / 2}(m), \\
& T_{4}=I_{3 / 2}(m), \quad T_{5}=I_{1 / 2}(n), \quad T_{6}=I_{3 / 2}(n), \\
& T_{7}=I_{1 / 2}(c), \quad T_{8}=I_{3 / 2}(c), \quad T_{9}=I_{3 / 2}\left(l \eta^{-1}\right) .
\end{aligned}
$$

\section{Acknowledgements}

The authors are thankful to the reviewers for their valuable suggestions and comments. One of the authors (Manpreet kaur) acknowledges MANF, UGC, India for providing the financial support (Grant No.: F1-17.1/2015-16/MANF-2015-17-CHH-66189/(SA-III/Website)). 


\section{References}

[1] Aero, E. L., Bulygin, A. N., Kuvshinskii, E. V., Asymmetric hydromechanics, Journal of Applied Mathematics and Mechanics 29 (1965) 333-346.

https://doi.org/10.1016/0021-8928(65)90035-3

[2] Aparna, P., Murthy, J. V. R., Rotary oscillations of a permeable sphere in an incompressible micropolar fluid, International Journal of Applied Mathematics and Mechanics 8 (2012) 79-91.

[3] Ariman, T., Turk, M. A., Sylvester, N. D., Applications of microcontinuum fluid mechanics, International Journal of Engineering Science 12 (1974) 273-293. https://doi.org/10.1016/0020-7225(74)90059-7

[4] Ashmawy, E. A., Rotary oscillation of a composite sphere in a concentric spherical cavity using slip and stress jump conditions, European Physical Journal Plus 130 (2015) 163. https://doi.org/10.1140/epjp/i2015-15163-8

[5] Condiff, D. W., Dahler, J. S., Fluid mechanical aspects of antisymmetric stress, Physics of Fluids 7 (1964) 842-854. https://doi.org/10.1063/1.1711295

[6] Eringen, A. C., Simple microfluids, International Journal of Engineering Science 2 (1964) 205-217. https://doi.org/10.1016/0020-7225(64)90005-9

[7] Eringen, A. C., Theory of micropolar fluids, International Journal of Mathematics and Mechanics 16 (1966) 1-18. https://doi.org/10.1512/iumj.1967.16.16001

[8] Happel, J., Brenner, H., Low Reynolds number hydrodynamics, Prentice-Hall, Englewood Cliffs, 1965.

[9] Iyengar, T. K. V., Vani, V. G., Oscillatory flow of a micropolar fluid generated by the rotary oscillations of two concentric spheres, International Journal of Engineering Science 42 (2004) 1035-1059. https://doi.org/10.1016/j.ijengsci.2003.10.006

[10] Kanwal, R. P., Slow steady rotation of axially symmetric bodies in a viscous fluid, Journal of Fluid Mechanics 10 (1) (1961) 17-24. https://doi.org/10.1017/S0022112061000020

[11] Keh, H. J., Lu, Y. S., Creeping motions of a porous spherical shell in a concentric spherical cavity, Journal of Fluids and Structures 20 (2005) 735-747. https://doi.org/10.1016/j.jfluidstructs.2005.03.005

[12] Lakshmana Rao, S. K., Bhujanga Rao, P., The oscillations of a sphere in a micropolar fluid, International Journal of Engineering Science 9 (1971) 651-672. https://doi.org/10.1016/0020-7225(71)90068-1

[13] Lakshmana Rao, S.K., Iyengar, T.K. V., Rotary oscillations of a spheroid in incompressible micropolar fluid, International Journal of Engineering Science 21 (1983) 973-987. https://doi.org/10.1016/0020-7225(83)90074-5

[14] Lamb, H., Hydrodynamics, Cambridge, Cambridge University Press, 1932.

[15] Lukaszewicz, G., Micropolar fluids: Theory and applications, Birkhuser, Basel, 1999. https://doi.org/10.1007/978-1-4612-0641-5

[16] Madasu, K. P, Gurdatta, M. K., Steady rotation of micropolar fluid sphere in concentric spherical container, Procedia Engineering 127 (2015) 469-475. https://doi.org/10.1016/j.proeng.2015.11.400

[17] Migun, N. P., On hydrodynamic boundary conditions for microstructural fluids, Rheologica Acta 23 (1984) 575-581. https://doi.org/10.1007/BF01438797

[18] Saad, E. I., Translation and rotation of a porous spheroid in a spheroidal container, Canadian Journal of Physics 88 (2010) 689-700. https://doi.org/10.1139/P10-040

[19] Sherief, H. H., Faltas, M. S., Saad, E. I., Slip at the surface of an oscillating spheroidal particle in a micropolar fluid, Australian and New Zealand Industrial and Applied Mathematics Journal 55 (2013) E1-E50. https://doi.org/10.21914/anziamj.v55i0.6813 
[20] Srinivasacharya, D., Iyengar, T. K. V., Rotary oscillations of an approximate sphere in an incompressible micropolar fluid, Indian Journal of Mathematics 43 (2001) 129-144.

[21] Srinivasacharya, D., Krishna Prasad, M., Slow steady rotation of a porous sphere in a spherical container, Journal of Porous Media 15 (12) (2012) 1105-1110. https://doi.org/10.1615/JPorMedia.v15.i12.30

[22] Stokes, G. G., On the effect of the internal friction of fluids on the motion of pendulums, Transactions of the Cambridge Philosophical Society IX(2) (1851).

[23] Tekasakul, P., Loyalka, S. K., Rotary oscillations of axi-symmetric bodies in an axi-symmetric viscous flow with slip: Numerical solutions for sphere and spheroids, International Journal for Numerical Methods in Fluids 41 (2003) 823-840. https://doi.org/10.1002/fld.467

[24] Tekasakul, P., Tompson, R. V., Loyalka, S. K., Rotatory oscillations of arbitrary axi-symmetric bodies in an axi-symmetric viscous flow: Numerical solutions, Physics of Fluids 10 (1998) 2797-2818. https://doi.org/10.1063/1.869803 